\title{
Interactive comment on "Cold season warming in the North Atlantic during the last 2000 years: Evidence from Southwest Iceland" by Nora Richter et al.
}

Nora Richter et al.

nora.richter@nioz.nl

Received and published: 4 November 2020

Please find our response to this review attached as a supplement.

Please also note the supplement to this comment:

https://cp.copernicus.org/preprints/cp-2020-84/cp-2020-84-AC3-supplement.pdf 